\section{Trimodality imaging of colonic lymphoma}

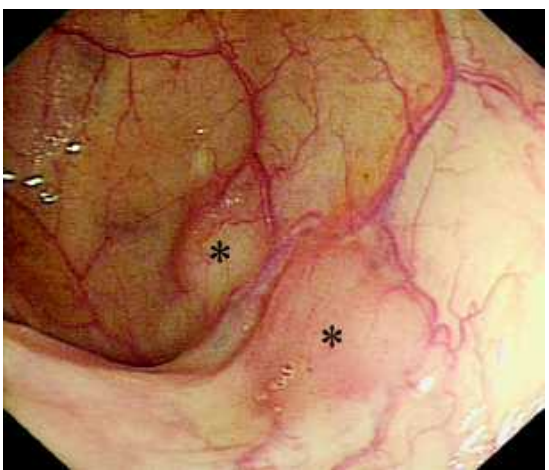

Fig. 1 Low-grade mucosa-associated lymphoid tissue lymphoma of the colon (asterisks; white-light endoscopy).

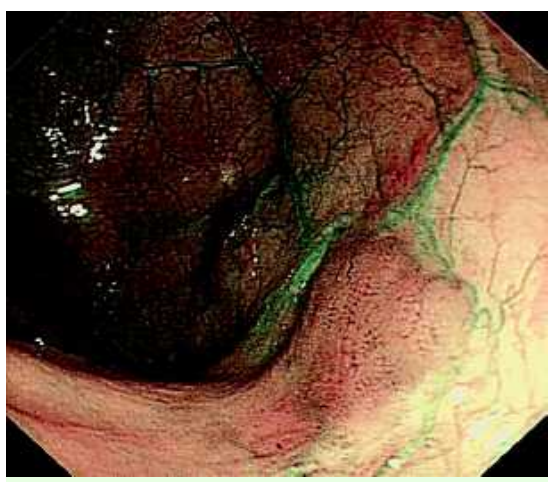

Fig. 2 Colonic lymphoma lesions enhanced by narrow-band imaging (the same view as in Fig. 1).

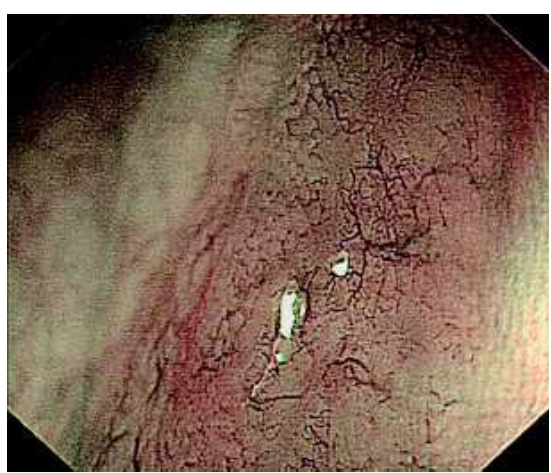

Fig. 3 Abnormal vascular pattern of lymphoma lesion under magnifying endoscopy (narrow-band imaging).

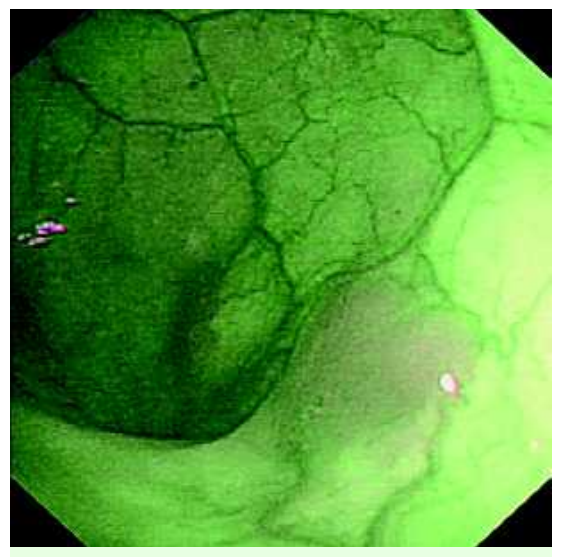

Fig. 4 Pale purple color of colonic lymphoma lesions under autofluorescence imaging (same view as in Fig. 1).
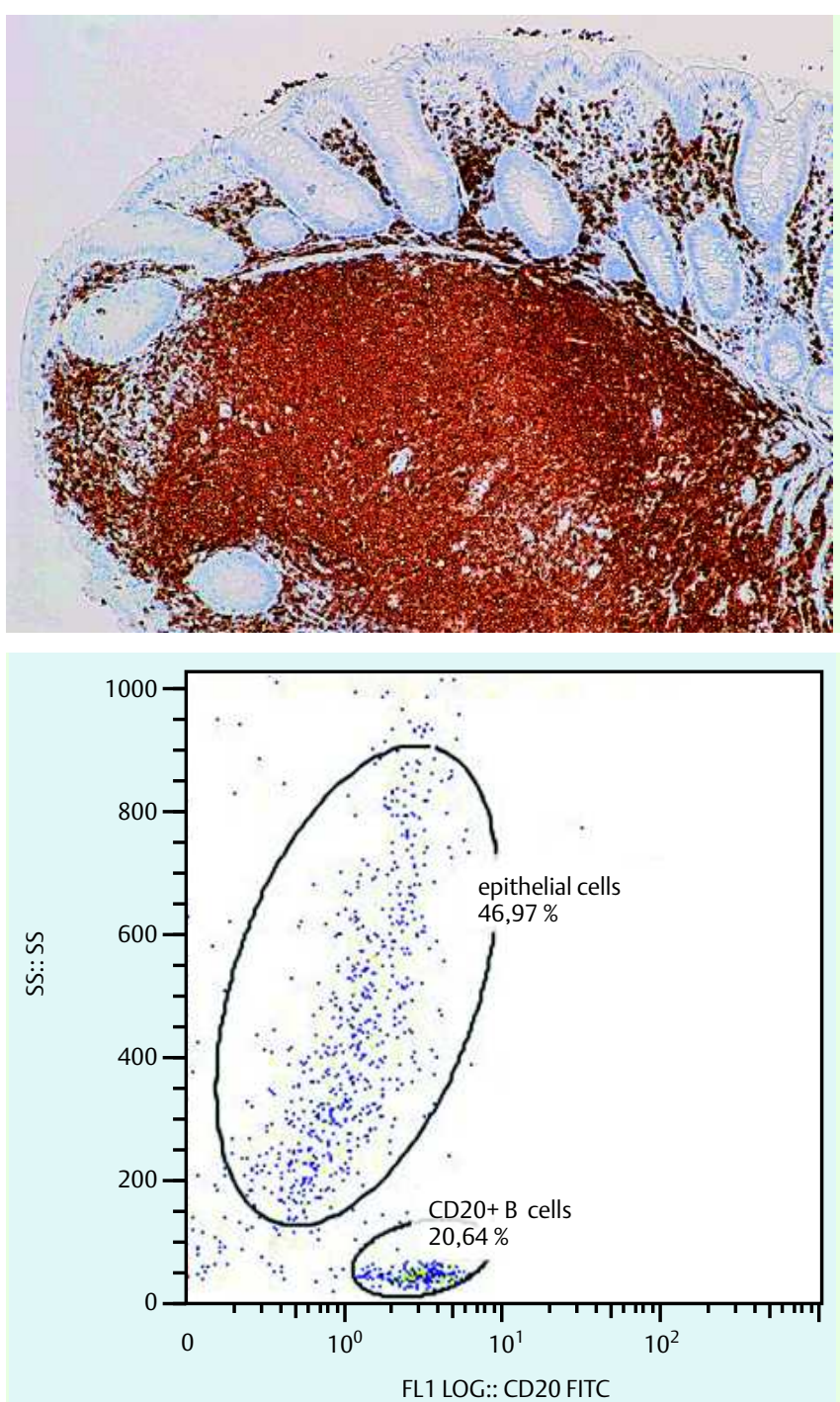

Fig. 5 Immunohistochemistry: infiltration of colonic submucosa and mucosa by small lymphoid cells of B-cell lymphoma (CD79- $\alpha$ staining).

Fig. 6 Flow cytometry. Colon biopsy was mechanically disintegrated. The malignant B lymphocytes were identified as a population of CD20-positive B cells. 
ic clone of B lymphocytes was detected in flow cytometry ( $\odot$ Fig. 6 ). To our knowledge this is the first report of trimodality imaging of colonic MALT lymphoma in the literature.

Primary colonic lymphoma is rare, but more thorough investigation might well lead to higher reported rates of gastrointestinal involvement [1]. Solitary or multiple protrusions are the most common pattern of MALT lymphoma in the colon [2]. Abnormal vessel pattern as a typical endoscopic feature of bowel lymphoma has been described previously by our group [3].

The term "trimodality imaging" refers to conventional white-light imaging assisted by narrow-band and autofluorescence imaging. Narrow-band imaging is able to enhance the visualization of tissue microvasculature, while autofluorescence imaging shows up reduced autofluorescence, and both these attributes can improve the detection of colonic neoplasia [4]. In conclusion, the dampened autofluorescence signal on autofluorescence imaging and abnormal vascular pattern on narrow-band imaging can represent important endoscopic features of lymphoma deposit in colonic mucosa. Trimodality imaging can thus facilitate the detection of this otherwise potentially discreet disease in the colon.

\section{Acknowledgment}

$\nabla$

The study was supported by research project MZO 00179906 from the Ministry of Health of the Czech Republic.

Endoscopy_UCTN_Code_CCL_1AD_2AC

\section{J. Cyrany ${ }^{1}$, M. Pintér ${ }^{1}$, V. Tyčová ${ }^{2}$, J. Krejsek ${ }^{3}$, D. Belada ${ }^{1}$, S. Rejchrt ${ }^{1}$, J. Bureš}

2nd Department of Internal Medicine, Charles University in Prague, Faculty of Medicine in Hradec Králové, University Teaching Hospital, Hradec Králové, Czech Republic

2 Fingerland Department of Pathology, Charles University in Prague, Faculty of Medicine in Hradec Králové, University Teaching Hospital, Hradec Králové, Czech Republic

3 Institute of Clinical Immunology and Allergology, Charles University in Prague, Faculty of Medicine in Hradec Králové, University Teaching Hospital, Hradec Králové, Czech Republic

\section{References}

1 Romaguera J, Hagemeister FB. Lymphoma of the colon. Curr Opin Gastroenterol 2004; 21: $80-84$

2 Saito T, Toyoda H, Yamaguchi M et al. Ileocolonic lymphomas: a series of 16 cases. Endoscopy 2005; 37: 466- 469

3 Kopáčová M, Rejchrt S, Tyčová V et al. Tumorous neo-vascularization: a newly recognized endoscopic feature of intestinal nonHodgkin lymphoma. Endoscopy 2007; 39 (Suppl 1): E93

4 Ponchon T. Colon tumors and colonoscopy. Endoscopy 2007; 39: 992 - 997

Bibliography

DOI $10.1055 / \mathrm{s}-2007-995696$

Endoscopy 2009; 41: E1 - E2

(c) Georg Thieme Verlag KG Stuttgart · New York . ISSN 0013-726X

Corresponding author

\section{J. Cyrany, MD}

2nd Department of Internal Medicine

Charles University Teaching Hospital

Sokolská 581

50005 Hradec Králové

Czech Republic

Fax: +420-495-834785

jiri.cyrany@email.cz 